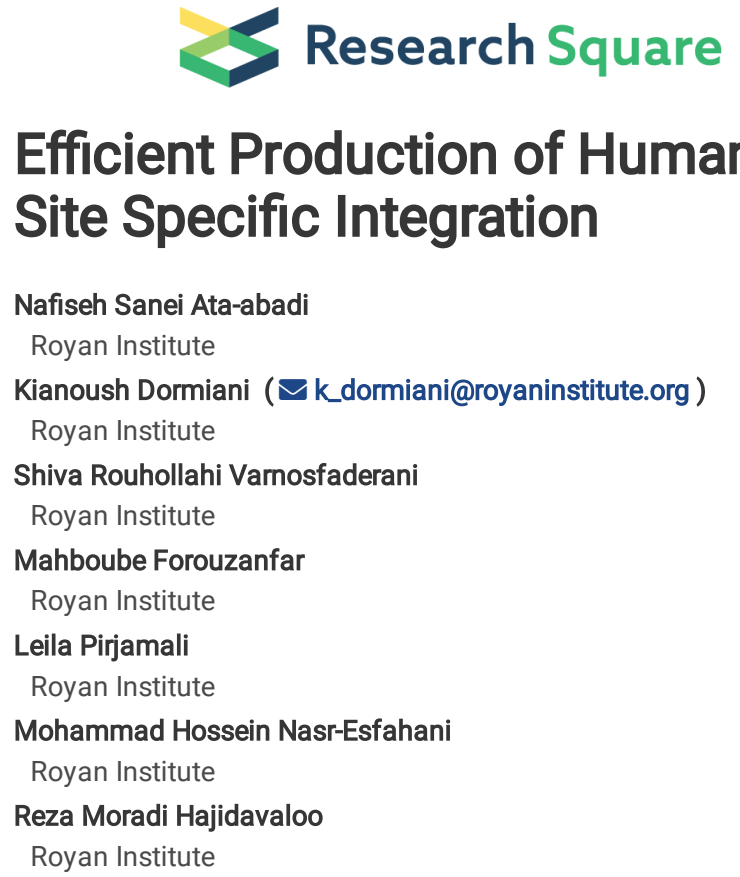

\title{
Efficient Production of Human Hyaluronidase PH20 in HEK293T Cells Using Site Specific Integration
}




\section{Abstract}

$\mathrm{PH} 20$ is hyaluronidase that hydrolyze the glycosidic bond of hyaluronic acid as a major proteoglycan found in extracellular matrices. $\mathrm{PH} 20$ is used in the subcutaneous space to increase the dispersion and absorption of co-administered drugs. $\mathrm{PH} 20$ is also injected against solid tumors for to better penetration of anticancer agents into the tumor tissue and inhibiting the tumor cell growth. In the present study, we have developed HEK293T stable cell lines secreting His-tagged human recombinant $\mathrm{PH} 20$ (rhPH20) in the culture supernatant through the PhiC31 integrase system. The produced rhPH20 was quantify using ELISA and turbidimetric assay and its activity was assessed through treatment of mouse cumulus-oocyte-complex (COCs). Furthermore, we have characterized genomic integration of $\mathrm{PH} 20$-containing vectors in one of the isolated clone with the highest levels of rhPH20 production. Our results demonstrated that the secreted rhPH20 in the culture supernatant contained a specific activity of approximately $3.5 \mathrm{IU} / \mathrm{ml}$ and it was properly able to denote all mouse oocytes. Consequently, it was revealed that $\mathrm{PH} 20$-expressing vectors integrated site-specifically in the PhiC31 pseudo attP sites in the host genome. Taken together, these results confirmed successful application of PhiC31 integrase as a robust approach for production of soluble, active rhPH20 in HEK293T cells.

\section{Introduction}

Hyaluronidases (Hyal) are members of a widespread group of enzyme family with limited ability to degrade chondroitin and chondroitin sulfates but preferentially hydrolyze hyaluronic acid as one of the most important components of extracellular matrix and basement membranes [1]. In human, hyaluronidase family consists of six members: HYAL1, HYAL2, HYAL3, HYAL4, HYALP1 and PH20 that are encoded through six genes clustered on two chromosomal regions, 7q31.3 and 3p21.3. Hyaluronidase PH20 (also known as sperm adhesion molecule 1; SPAM1) is generally attach to the sperm plasma membrane via a glycosylphosphatidyl inositol (GPI) anchor and whose major function is helping sperm to penetrate through hyaluronic acid-rich cumulus layer around the oocyte [2, 3]. Translation of human PH20 mRNA results in a 509-amino acid precursor protein consist of a signal sequence with 35 amino acids at the $\mathrm{N}$-terminus and a mature $\mathrm{PH} 20$ polypeptide with 474 amino acids. Expression of mature $\mathrm{PH} 20$ polypeptide lacking $\mathrm{GPI}$-attachment sites results in production of the soluble form of human $\mathrm{PH} 20$ [4]. There are several approved therapeutics applications for recombinant human $\mathrm{PH} 20$ (rhPH20). For example, $\mathrm{rhPH} 20$ can be utilized as an adjuvant in subcutaneous fluid administration for achieving hydration, increasing the absorption and bioavailability of the subcutaneous drugs through the degradation of hyaluronic acid and enhancing the permeability of connective tissues [5-7]. Hyaluronidase is also indicated for increasing resorption of radiopaque agents in subcutaneous urography [4]. On the other hand, rhPH20 can also be used for dispersion of cumulus-oocyte complex (COCs) before intracytoplasmic sperm injection (ICSI) in order to visualize, grade, and manipulate the target oocyte [8]. Up to now, rhPH20 is produced successfully using various expression systems including Drosophila Schneider-2 (DS-2), Chinese Hamster Ovary (CHO) cells, Pichia pastoris and Arabidopsis thaliana [4, 8-11]. Mammalian cells, especially human cell lines are preferred for expression of recombinant human proteins with complex post-translational modifications and protein folding. Among human cell lines, HEK293T cell line is an interesting expression system because of its ability to establish human glycosylation profiles and high yield of recombinant protein expression [12]. Traditional methods for generation of stable cell lines depend on random integration(s) of the transgene that lead to unsteady and unpredictable protein expression. Site-specific recombination systems are powerful methods for inserting the transgene, which leads to long term and high levels of recombinant protein expression [13]. Among site-specific recombinases, PhiC31 serine integrase mediates specific unidirectional integration of the donor plasmid containing transgene in the host genome compared to reversible recombination through bacteriophage recombinases such as Cre and Flp $[14,15]$. PhiC31 mediates recombination between bacterial attachment site (attB) of donor plasmid and pseudo phage attachment sites (pseudo attP) in the host genome of mammalian cells, which finally generate new hybrid sites, attR and attL with different sequences from attB and attP [16-18]. An interesting feature of PhiC31 integrase is that the enzyme prefers transcriptionally active, open chromatin domains for targeted integration. This feature results in a higher rate and uniform transgene expression [19, 20].

Herein, we reported the development of transgenic HEK293T stable clones contain non-viral vector that expressing rhPH20. For this purpose, HEK293T cells were co-transfected with constructed donor vector and the plasmid-encoding PhiC31 integrase. Following isolation and expansion of transgenic clones, concentration, activity and functionality of the active $\mathrm{rPH} 20$, which is purified from the culture supernatant of individual clones were evaluated. Besides, the copy number of $\mathrm{PH} 20$ donor vector and its integration sites in the genome of the stable recombinant clones were determined.

\section{Materials And Methods}

\section{Design and construction of expressing vectors}

Amplification of His-tagged PH20 was performed in two PCR steps. First, Human PH20 coding sequence (GenBank: BC026163.1) free from glycosyl phosphatidyl inositol anchor was amplified using specific primers F-PH20 (5'-CATAGTCATTACTCTTTACAATGGGAG-3') and R-PH20 (5'-

CTAACAATGAACATTGTGGCAGATAG-3') from human testis cDNA. At the second step, a fragment containing His-tag and mouse IgGK signal peptide was added to the upstream of amplified $P H 20$ coding sequence using specific primers F-PHEF1 (5'GGTACCGAGATGGAGACAGACACACTCCTGCTATGGGTACTGCTGCTCTGGGTTCCAGGTTCCACTGGTGACCATCATCACCATCACCATGACGACGACGACAAGCTGA^ $3^{\prime}$ ) and R-PHEF (5'-CTCGAGTTAGTAGAAAATTTGAGGTTCTTCTGTCTCCATG-3'). Amplified His-PH20 sequence was sub-cloned into Kpnl and Xhol sites of pBudCE4.1 (Invitrogen, USA), under the control of EF-1区 promoter to generated transient recombinant vector pBud.PH20. Afterward, attB sequences was amplified from plasmid pDB2 as the template and sub-cloned into Nhel site of pBud.PH20 to generate plasmid pBud.PH20.attB. In order to prepare pBud.2xPH20.attB, the fragment containing His-PH20 was sub-cloned into Sall and Xbal sites of pBud.PH20.attB, downstream of CMV promoter to construct pBud.2xPH20.attB vector. On the other hand, the puromycin resistance gene expression cassette was amplified from pTRE2pur plasmid followed by bluntending by Klenow fragment and sub-cloned into Pvul site of pBud.2xPH20.attB plasmid, which was blunt ended previously. The sub-cloned sequences of His$\mathrm{PH} 20$, attB and puromycin resistance cassette in the structure of final expression vector were verified by Sanger sequencing. All restriction enzymes were purchased from Thermo Scientific Company. The PhiC31 integrase expression plasmid, pCMV-Int, was generously gifted by Prof. Michelle Calos (Stanford University, USA). 
HEK293T cell line was obtained from Pasteur Institute of Iran (Tehran, Iran). The cells were grown and maintained in high glucose DMEM (Gibco, USA) supplemented with $10 \%$ fetal bovine serum (FBS), 100 units $/ \mathrm{ml}$ penicillin and $100 \mu \mathrm{g} / \mathrm{ml}$ streptomycin sulfate (Gibco, USA) at $37{ }^{\circ} \mathrm{C}$ and $6 \% \mathrm{CO}_{2}$. $\mathrm{HEK} 293 \mathrm{~T}$ cells were co-transfected with the pBud.PH20.attB vector and pCMV.Int at a mass ratio of 1:40 using Lipofectamin 2000 (Thermo scientific, USA) according to the manufacturer's instructions. After $24 \mathrm{~h}$ of transfection, cells were split into $10 \mathrm{~cm}$ plates and incubated for $48 \mathrm{~h}$. Afterward, cells were selected in medium containing $60 \mu \mathrm{g} / \mathrm{ml}$ zeocine (InvivoGene, USA) for 2-3 weeks at which time antibiotic-resistant clones became observable. Each clone was picked using a pipette tip and transferred to an individual well of a 24-well plate and subsequently expanded for further analysis. The best clone with the highest PH20 expression level and activity was chosen for second round of co-transfection with the PBUD.2xPH20.attB vector and pCMV.Int. The process of stable cell line development was the same as mentioned for PBUD.PH20.attB. However, in this round the new recombinant clones were selected in medium containing puromycin with a final concentration of $1 \mu \mathrm{g} / \mathrm{ml}$.

\section{Production of the recombinant PH20 in HEK293 cells}

Stably transfected HEK293T cells were expanded and maintained in T-150 flasks (TPP, Switzerland) containing 30 ml high glucose DMEM supplemented with $10 \% \mathrm{FBS}$ and $1 \mathrm{mg} / \mathrm{ml}$ puromycin at $37{ }^{\circ} \mathrm{C}$ and $6 \% \mathrm{CO}_{2}$. After reaching cell confluence (2-3 days) the medium supplemented with $5 \% \mathrm{FBS}$ in the absence of puromycin was refreshed. Cells were maintained for three days at mentioned medium and subsequently the supernatants were harvested. Replacing of fresh medium followed by harvesting the supernatant was performed for two additional rounds.

\section{Assessment of recombinant PH20 concentration using ELISA}

Concentration of secreted His-tagged rhPH20 was determined using human hyaluronidase PH20 (SPAM1) ELISA kit (CUSABIO) according to the manufacturer's instructions. Briefly, a serial dilutions of different concentrations of PH20 were provided using standard solution. Then, coated microwells with $\mathrm{PH} 20$ specific antibody were incubated with $100 \mu \mathrm{l}$ of standard dilutions and culture supernatants from HEK293T clones expressing rhPH20 for $2 \mathrm{~h}$ at $37^{\circ} \mathrm{C}$. Next, unbound substances were removed and a biotin-conjugated antibody specific for $\mathrm{PH} 20$ was added to each well and the plate was incubated for $1 \mathrm{~h}$ at $37^{\circ} \mathrm{C}$. The wells were washed and avidin-conjugated Horseradish Peroxidase (HRP) was added to the each well and was incubated for $1 \mathrm{~h}$ at $37^{\circ} \mathrm{C}$.

Subsequently, TMB Substrate was added to the wells to color development in proportion to the amount of rhPH20 in the supernatant and standards. Optical density (OD) of each well was measured at $450 \mathrm{~nm}$ using a spectrophotometric plate reader (Stat Fax 3200, Vesta Tajhiz Part, Iran). Finally, a standard curve was provided and concentration of secreted $\mathrm{PH} 2 \mathrm{O}$ in supernatant was determined using this standard curve.

\section{Assessment of recombinant $\mathrm{PH} 20$ specific activity using turbidimetric assay}

The enzymatic activity of secreted His-tagged rhPH2O in each culture supernatant was also measured using microturbidity assay described by Rapport et al. [35]. This method is based on the generation of an insoluble precipitate by the $\mathrm{PH} 20$ enzyme when acidified serum albumin interacts with hyaluronic acid. First, a working solution of $6 \mathrm{U} / \mathrm{ml}$ hyaluronidase (Sigma, H3506) was prepared in enzyme diluent (20 mM sodium phosphate with $77 \mathrm{mM}$ sodium chloride and $0.01 \%(\mathrm{w} / \mathrm{v})$ bovine serum albumin, $\mathrm{pH} 7.0$ at $37^{\circ} \mathrm{C}$ ). In addition, hyaluronidase serial dilutions and culture supernatant were prepared in enzyme diluent. Next, $1 \mathrm{ml}$ of hyaluronic acid solution [0.03\% (w/v)] was added to all tubes followed by incubating for $45 \mathrm{~min}$ at $37^{\circ} \mathrm{C}$. In the next step, $0.5 \mathrm{ml}$ of each tube was transferred into suitable cuvettes containing $2.5 \mathrm{ml}$ of acidic albumin and transmittance percent of each cuvette was evaluated at $600 \mathrm{~nm}$. Finally, the activity of secreted rhPH20 in culture supernatant was determined according to standard curve of hyaluronidase serial dilutions.

\section{Monitoring the stability of recombinant clone}

To investigate the stability of selected recombinant clone, whose expression of rhPH20 was checked for 16 weeks. For this, the cells of selected clone and untransfected cells as the negative control were cultured in 12-well plate in the same condition mentioned previously in the absence of puromycin selective pressure. After maintaining the cells in the culture medium for three days, the supernatant was isolated and the concentration of secreted rhPH20 was measured in each specific time points during a 16-week monitoring period using human hyaluronidase PH20 ELISA kit (CUSABIO) according to the manufacturer's instructions.

\section{Purification of the recombinant $\mathrm{PH} 20$}

Secreted His-tagged rhPH20 in the medium was purified using HIS-SelectR Nickel Affinity Gel (Sigma, USA) as previously described [34]. Briefly, supernatant was diluted twice by adding $50 \mathrm{mM}$ Tris-HCl, pH 7.5, $500 \mathrm{mM} \mathrm{NaCl}$ and $10 \mathrm{mM}$ imidazole. Ni-NTA resin (HIS-SelectR Nickel Affinity Gel (Sigma, USA) mixed with media and rolled over the bottle at $4{ }^{\circ} \mathrm{C}$ overnight ( $1 \mathrm{ml} \mathrm{Ni-NTA}$ resin for $100 \mathrm{ml}$ medium) and then centrifugied at $5000 \mathrm{~g}$ for $5 \mathrm{~min}$. Remained resin-PH20 complex was washed twice with washing buffer (Tris-Hcl 50 mM, pH 7.5, Imidazole 20 mM, NaCl 500 mM ). Finally, His-tagged rhPH20 was eluted using elution buffer (Tris-Hcl 50 mM, pH 7.5, NaCl 500 mM and Imidazole $250 \mathrm{mM}$ ).

\section{Western blot}

For western blotting, purified His-tagged rhPH20 was separated using 12\% SDS-PAGE gel electrophoresis followed by electro-transferring to a Polyvinylidene fluoride (PVDF) membrane. The skim milk-blocked membranes were probed using Anti-polyHistidin peroxidase (Sigma; A7058; 1:500) as the primary antibody and HRP-conjugated goat anti-mouse IgG (Dako; P0447; 1:5000) as the secondary antibody. Subsequently, detection of HRP-conjugated IgG bound to protein band was performed using ECL Advance Western Blotting Detection Kit (Amersham Biosciences, RPN2135) according to the instruction of the manufacturer.

Evaluation of recombinant $\mathrm{PH} 20$ activity by cumulus cell removal 
This study was approved by the Institutional Review Board of the Royan Institute. Also all animal experiments were performed in accordance with the ethical guidelines established by the Institutional Ethical Committee of the Royan Institute, which are in compliance with the ARRIVE guidelines. For evaluating the functionality of rhPH20, female mice were superovulated with $10 \mathrm{IU}$ of pregnant mare's serum gonadotropin (PMSG) (Pregnocol, Australia) on day 1, followed by $10 \mathrm{IU}$ of human chorionic gonadotropin (hCG) (Pregnyl, Iran) on day 3. $18 \mathrm{~h}$ after the hCG injection both oviducts were removed and flushed for collection of mouse COCs. Animals were euthanized at the end of this stage with dislocation of cervical vertebra. Then, COCs treated with 25 ul culture supernatant containing $\mathrm{rhPH} 20(3.5 \mathrm{IU} / \mathrm{ml})$ and $25 \mathrm{ul}$ commercially available hyaluronidase (300 IU/ml, Sigma, H3506) as control. The time of cumulus cell removal were compared between two groups. In parallel, for toxicity test of hyaluronidase, mouse embryo assay was carried out. Briefly, female mice were superovulated with 10 IU of pregnant mare's serum gonadotropin (PMSG) (Pregnocol, Australia) on day 1, followed by 10 IU of human chorionic gonadotropin (hCG) (Pregnyl, Iran) on day 3. After hCG injection, each female mouse was placed in an individual cage overnight with a mature NMRI male mouse with proven fertility. The next morning, successful mating was confirmed by the presence of a vaginal copulation plug. The appearance of a vaginal plug was designated as first day of the pregnancy. Female mice with a vaginal plug were sacrificed after $21 \mathrm{~h}$ posthCG injection and both oviducts were removed and flushed for collection of 2PNs. 2PNs were treated for $30 \mathrm{~min}$ by both supernatant containing rhPH20 and commercially available hyaluronidase and cultured in KSOM for 5 days. At last, cleavage and blastocysts rates were evaluated. A phase contrast stereomicroscope (Olympus, SZX12, Japan) were utilized for imaging.

\section{Transgene copy number determination using quantitative PCR}

Genomic DNA was isolated from several puromycin-resistant clones using DNeasy blood and tissue kit (Qiagen, Germany) according to the manufacturer's instructions. Absolute copy number of PH20 expressing vector integrated into the target genome was measured using quantitative PCR )qPCR( [36]. To generate the external standard curve, a serial dilution of human genomic DNA containing 1, 5, 25125 and 625 copies of the PH20 cDNA were prepared. The mass of human genomic DNA contains one copy of the $\mathrm{PH} 20 \mathrm{cDNA}$ was calculated according to the following equation:

$[\mathrm{n}][1.096 \mathrm{e}-21 \mathrm{~g} / \mathrm{bp}]$

1 copy PH20 per haploid genome

in which $\mathbf{n}$ is the size of human genome (3.4e9bp, haploid) and $\mathbf{1 . 0 9 6 e - 2 1}$ is the mass of $1 \mathrm{bp}$. Quantitative PCR was performed in a Step One Plus Real-Time PCR thermal cycler (Applied Biosystems, USA) using SYBR master mix (TaKaRa, Japan). All reactions were performed in triplicate using specific primers for human PH20 cDNA (F-rhPH20: TTCAGAGCACCTCCTGTTATTC and R-rhPH20: GGCTCATATCTAGTGGCTCATC). To estimate the copy number of PH20 transgene, two dilutions (40 and $8 \mathrm{ng} / \mu \mathrm{l}$ ) of the genomic DNA were used as the template for PCR reactions.

\section{Rescue and analysis of integration sites}

The precise locations of the integrated plasmids carrying PH20 in the genome of HEK293T transgenic clones were determined using inverse PCR method. Genomic DNA samples were digested with Spel and Xbal restriction enzymes (Thermo Scientific, USA) followed by precipitation using ethanol. After that, selfcircularization of the digested genome was carried out using T4 DNA ligase (Thermo Scientific, USA) and purified during ethanol precipitation process. Resulted DNA samples were utilized as templates for the first round of inverse PCR using Smar Taq DNA polymerase (Cinnagen, Iran) with specific primers (FP1: CCCCATGGCTCCGACCGAAG and R-P1: ACGTCCCGTGCTCACCGTGAC). Product of the first PCR was employed as template of the second round of inverse PCR as a semi-nested PCR with the same forward primer (F-P1: CCCCATGGCTCCGACCGAAG) and an internal reverse primer (R-P2:

TGGACCAGATGGGTGAGGTGGAGTAC). The final inverse PCR products were loaded on $1 \%$ gel agarose followed by purification by a QIAquick Gel Extraction Kit (Qiagen, Germany). Purified bands were T/A cloned into the pUCM-T vector (Bio Basic, Canada) and sequenced with M13 universal primers. The sequence results were aligned with the reference human genome, UCSC genome browser (GRCh37/hg19) to detect the precise genomic locations [37].

\section{Statistical analysis}

Statically analysis was performed by one-way ANOVA using SPSS (Version 21, USA). The values are represented as mean (s.e.m.) or mean (s.d.) for three independent repeats in each experiment and the values at $\mathrm{P}<0.05$ were considered significant.

\section{Results}

\section{Expression vectors}

We designed and constructed two vectors encoding human PH20 including pBUD.PH20.attB and pBUD.2xPH20.attB (Fig. 1). These two vectors composed of different genetic elements; First, the Pbud.ph20.attB vector was developed that contained four major parts including: (i) the human PH20 coding DNA sequence (CDS) under the control of Human elongation factor-1 alpha (EF-1a) promoter, (ii) the bleomycin resistance gene that BleoR confers resistance to zeocin to select preliminary clones (iii) the attB element as specific sequence recognized by PhiC31 integrase and (iv) the bacterial backbone. Afterward, the pBUD.2xPH20.attB vector was developed with the pBUD.PH20.attB as template vector with two main changes: (i) inserting the second human PH20 CDS downstream of $\mathrm{CMV}$ promoter and (ii) addition of a cassette containing puromycin resistance gene (pur') to select ultimate stable clones. Both $\mathrm{PH} 20$ expression vectors were co-transfected with PhiC31 integrase encoding vector, pCMV-Int into the HEK293T cells.

\section{Transfection and clonal selection based on recombinant $\mathrm{PH} 20$ concentration}

Generation of stable rhPH20 expressing clones were achieved through two rounds of transfection. First, HEK293T cells were transfected with pBUD.PH20.attB and pCMV.INT plasmids. Screening of these cells with zeocin resulted in several transgenic clones. Preliminary experiments showed that 5 clones expressed the highest levels of rhPH2O in culture supernatant. With more screening among them, the clone with the highest expression rate of rhPH20 (named HEK- 
PH20-1) was selected by means of human PH20 ELISA kit (Fig. 2A). The HEK-PH20-1 clone was retransfected with pBUD.2xPH20.attB. Selection of the transfected cells with puromycin finally results in 3 clones, which among them the $\mathrm{C}-1 \mathrm{~A}$ clone showed the maximum expression rate of rhPH20 in the culture supernatant using human PH20 ELISA kit (Fig. 2B).

\section{Specific activity of secreted recombinant $\mathrm{PH} 20$}

The specific activity of His-tagged rhPH2O in the culture supernatant of C-1A clone with the highest rhPH20 expression rate (named HEK-PH20-1A) was calculated via turbidimetric assay. This test was carried out based on a standard curve, which was plotted using commercially available PH20 from bovine testis. Resulted standard curve was prepared by measuring the activity of seven dilutions of commercially available PH20 from 1 to $4.5 \mathrm{IU} / \mathrm{ml}$ had $\mathrm{R}^{2}$ value of 0.9984. The results of turbidimetric analysis confirmed that the maximum activity of His-tagged rhPH20 in culture supernatant of HEK-PH20-1 A clone was 3.5 $\mathrm{IU} / \mathrm{ml}$. Conversely, there was almost no activity of PH20 in the supernatant of untransfected HEK293T culture employed as the negative control. (Table 1).

\begin{tabular}{|c|c|c|c|c|c|c|c|c|c|}
\hline Sample & $\mathrm{T} 1^{*}$ & T2 & T3 & T4 & T5 & T6 & T7 & HEK-PH20-1A & HEK293T" \\
\hline Absorbance $(600 \mathrm{~nm})$ & -0.0795 & -0.0391 & -0.0004 & 0.0410 & 0.0931 & 0.1688 & 0.6003 & 0.0004 & 0.6001 \\
\hline Transmittance (\%) & 120.11 & 109.42 & 100.14 & 90.93 & 80.70 & 67.84 & 25.13 & 100.10 & 25.11 \\
\hline Unit (IU/ml) & 4.51 & 3.99 & 3.52 & 3.02 & 2.49 & 2.01 & 0.00 & 3.51 & 0.00 \\
\hline \multicolumn{10}{|c|}{ * T1-T7: Serial dilutions of commercially available bovine PH20 } \\
\hline \multicolumn{10}{|c|}{ \# HEK293T: Untransfected HEK293T culture supernatant } \\
\hline
\end{tabular}

\section{Stability of the selected recombinant clone}

To analysis the stability of recombinant clone expressing rhPH20, the expression level of rhPH2O in culture supernatant of both HEK-PH20-1A cells and untransfected HEK293T cells as negative control, were assessed during 16 weeks using ELISA assay. The results demonstrated that expression rate by HEK$\mathrm{PH} 20-1 \mathrm{~A}$ not only was strongly higher than that of the control cells, but also did not change significantly during selected time points in the 16-week monitoring period (Fig. 3).

\section{Purification and analysis of recombinant $\mathrm{PH} 20$ using western blot}

In order to purification of secreted His-tagged rhPH20, The HEK-PH20-1A culture supernatant was harvested after three days incubation followed by purifying of His-tagged rhPH20 by nickel resin affinity purification. Approximately $200 \mu \mathrm{g}$ of rhPH 20 was yielded from $1 \mathrm{~L}$ of culture supernatant. Analyzing the isolated protein using western blot showed an apparent $58 \mathrm{kDa}$ band appeared on the membrane using anti-polyHistidin peroxidase (Fig. 4).

\section{The effects of recombinant PH20 on the removal of cumulus cells}

Mouse COCs were treated with culture supernatant containing $\mathrm{hPH} 20$ and commercially available hyaluronidase as a control to remove the cumulus cells from oocytes. Results showed that all of the oocytes were denuded after treatment with filtered culture supernatant containing rhPH2O during the same period with the control. In addition, the grading of resulting blastocysts treated with rhPH20 were similar to the control group (Fig. $5 \mathrm{~A}$ ). Moreover, mouse $2 \mathrm{PNs}$ were exposed $30 \mathrm{~min}$ to assess prolonged rhPH20 treatment on resulting embryo. Developmental rate also showed no significant differences between the experimental groups (Fig. 5B, C).

\section{Transgene copy number estimation}

The transgene copy number was estimated using quantitative PCR (qPCR). The absolute quantitative standard curve was prepared and showed a correlation coefficient of 0.9992 and PCR efficiency was determined to be $107 \%$ (slope $=-3.160$ ). Additionally, the PCR product specificity was confirmed by melting curves analysis. These data confirmed that the method is optimized for absolute quantification of transgenic $\mathrm{PH} 20$ copy number. Considering two copies of endogenous $\mathrm{PH} 20$ in normal human genome, the copy number of $\mathrm{PH} 20$ transgene was estimated, three copies in clones 1 and 6 , and five copies in clones $1 \mathrm{~A}$ and $1 \mathrm{C}$. According to these results, it can conclude that a single copy of pBUD.PH20.attB (one-copy donor vector) and a single copy of pBUD.2xPH20.attB (two-copy donor vector) have integrated in the genome of clones C-1 A and C-1C that were transfected twice. In the case of clones C-1 and C-6, which were transfected once, evidence demonstrated that a copy of pBUD.PH20.attB was integrated in each of these clones (Table 2). 


\begin{tabular}{|c|c|c|c|c|c|}
\hline Cells & Quantity (ng) & Ct value $\pm S D^{1}$ & $\begin{array}{l}\text { Transgene/ } \\
\text { endogenous ratio }\end{array}$ & Total PH20 copy per cell & Rounded transgene copy per cell \\
\hline \multirow[t]{2}{*}{ NBC } & 2 & 26.82 & 0.00 & 2.00 & 0 \\
\hline & 8 & 24.46 & 0.00 & 2.00 & 0 \\
\hline \multirow[t]{2}{*}{ HEK293T } & 2 & $26.77 \pm 0.12$ & 0.00 & 2.00 & 0 \\
\hline & 8 & $24.35 \pm 0.06$ & 0.00 & 2.00 & 0 \\
\hline \multirow[t]{2}{*}{ C-1A } & 2 & $25.54 \pm 0.08$ & 2.46 & 4.92 & 3 \\
\hline & 8 & $23.14 \pm 0.05$ & 2.4 & 4.81 & 3 \\
\hline \multirow[t]{2}{*}{ C-1C } & 2 & $25.44 \pm 0.25$ & 2.64 & 5.28 & 3 \\
\hline & 8 & $23.04 \pm 0.05$ & 2.59 & 5.19 & 3 \\
\hline \multirow[t]{2}{*}{ C-1 } & 2 & $26.24 \pm 0.14$ & 1.47 & 2.95 & 1 \\
\hline & 8 & $23.87 \pm 0.09$ & 1.41 & 2.83 & 1 \\
\hline \multirow[t]{2}{*}{ C-6 } & 2 & $26.25 \pm 0.15$ & 1.46 & 2.92 & 1 \\
\hline & 8 & $23.81 \pm 0.04$ & 1.48 & 2.96 & 1 \\
\hline
\end{tabular}

\section{Identification of vector integration sites}

The extracted genome of HEK-PH20-1A colon with the highest expression level of rhPH20 in culture supernatant, was used to determine the specific genomic sites that pBud.2xPH20.attB and pBud.PH20.attB were integrated. First, the complete sequence of attB in donor vectors was amplified in the extracted genome of HEK-PH20-1 A clone, un-transfected HEK293T as the negative control and pBud.2xPH20.attB plasmid as a positive control. Our results demonstrated that PhiC31 catalyzed the site specific recombination between attB site in the donor vectors and the pseudo attP sites in HEK-PH20-1A genome, since the expected band of attB sequence with the length of $140 \mathrm{bp}$ was not amplified (Fig. 6A). Afterward, donor vector integration sites were identified in the genome of HEKPH20-1 A colon, using a nested inverse PCR. In this method, digested genome of experimented colon with Xbal and Spel was self-circularized and used as PCR template. We employed a two-step PCR (primers F-P1 and R-P1 for the first round of PCR; primers F-P1 and R-P2 for the second round of PCR) for amplification of un-known sequence adjacent to known sequence of integrated donor vector. Results of electrophoresis revealed three bands with the size of approximately 300, 900 and 2000 bp (Fig. 6B). Fig. 6C shows the Spel and Xbal sites located on the integrated vectors and used to digest the transgenic genome of the target clone. Moreover, the location of primers used for inverse PCR have also been determined on the map of donor vectors before site specific integration in Fig. 6C. Fig. 6D depicts the location of inverse PCR primers on the map of donor vectors after site specific integration.

BLAST analysis of the inverse PCR band sequences revealed two recombination events on the chromosomal regions of 10q21.2 and $20 \mathrm{q} 11.22$ in the genome of HEK-PH20 1A clone. These results also indicated that recombination reactions between attB site of pBud.2xPH20.attB and pBud.PH20.attB and the pseudo attP sites on these regions were along with small deletions ( $\leq 22$ bp) (Fig. 7).

\section{Discussion}

Previous studies demonstrated the safety issue of testicular hyaluronidase in clinical applications as a disadvantage. This is due to the presence of endotoxin and other proteins that lead to inflammatory responses [21, 22]. Therefore, recombinant human $\mathrm{PH} 20$ is preferred to animal testis-derived hyaluronidase for therapeutics usage, because of its safety, purity, potency, effectiveness and human-origin [8]. In the present study, we successfully constructed two expression vectors pBud.PH20.attB and pBud.2xPH20.attB carrying one and two copies of human His-tagged PH20 expression cassette, respectively. Taking advantage of PhiC31 integrase system, after two rounds of transfection of these two donor vectors together with pCMV-Int, several stable cell lines expressing rhPH20 were obtained. The quantitative analyzing of secreted $\mathrm{PH} 20$ in culture supernatant of various isolated clones gives rise to the identification one clone named HEK-PH20-1A that showed the highest expression level of rhPH20. The results of turbidimetric assay demonstrated that the rhPH20 specific activity in HEK$\mathrm{PH} 20-1 \mathrm{~A}$ culture supernatant reached to a maximum of $3.5 \mathrm{U} / \mathrm{ml}$. Up to now, the recombinant hPH20 were successfully expressed in several expression systems including plant expression system such as Arabidopsis thaliana [11] and Nicotiana benthamiana [23], yeast expression systems such as Pichia pastoris [9] and mammalian expression systems such as Chinese Hamster Ovary (CHO) cells [4]. Due to presence of several potential $\mathrm{N}$-linked glycosylation sites on human $\mathrm{PH} 20$ and their importance in optimal activity of the enzyme [4], we aimed to express rhPH20 in an appropriate cell line to provide human glycosylation pattern for our produced $\mathrm{rPH} 2 \mathrm{O}$. $\mathrm{CHO}$ cells are among the most frequently used cells for production of recombinant biotherapeutic proteins. However, they don't have ability to produce some types of human glycosylation, but instead produced non-human glycan structures that may lead to immunogenicity in human $[24,25]$. Besides, our pervious study showed that adherent $\mathrm{CHO}$ cells are not appropriate host cell for rhPH20 production. In this experiment, all the cells transfected with rhPH2O producing vector were extensively destroyed during about $8 \mathrm{~h}$ post-transfection due to the destructive effect 
of the cell culture-secreted enzyme (data not shown). Accordingly, we decided to choose a human cell line, HEK293T cells to develop a human based expression system using site-specific recombination by PhiC31 integrase for efficient production of rhPH20. There are many benefits of using HEK cells, namely, the cells are semi-adherent cells that are divide rapidly with proper posttranslational modifications especially protein folding and glycosylation [24, 26]. They are employed for both transient and stable protein expression, and can be cultured in both suspension and a monolayer. HEK cells are transfected easily with a variety of methods and have the capacity of a cell factory for manufacturing a large amount of recombinant proteins [27, 28]. The important finding in our experiments was that the expressed rhPH2O could not cause damage of HEK cells due to this fact that the cells are semi-adherent in comparison to adherent $\mathrm{CHO}$ cells. One of the major obstacles of stable expression of recombinant proteins in eukaryotic cells is the variable and unstable protein production patterns, which is mostly due to position effect of transgene integration site arising from random integration of the donor vector [29, 30]. Sitespecific integration of transgene could be an ideal system for long-term of recombinant proteins in eukaryotic cells [31]. Phic31 is an efficient recombinase that catalyzes a unidirectional integration between attB site in the donor vector and pseudo attP sites in human genome that make it a powerful system for targeting expression of transgene in various types of cells [32]. Using this system, a 60-fold increasing in luciferase expression was obtained compared to random integration [331]. By combination of HEK293T as a human expression system with PhiC31 site-specific integration, we were able to achieve approximately $200 \mu \mathrm{g} \mathrm{rhP} 20$ in $1 \mathrm{~L}$ of culture medium of HEK-PH20-1A colon. Another important problem in developing of the stable cell lines for manufacturing recombinant proteins is reducing the rate of protein production over time.

One of the key applications of rhPH20 is the removal of the COCs containing cumulus granulosa cells surrounding the oocyte prior to ICSI [8]. In the present study, we assessed the activity of the secreted rhPH20 in culture supernatant of HEK-PH20-1A colon via treatment of mouse COCs that leads to successful denuded oocytes and derived embryos with high quality. The obtained results were comparable with commercially available testicular hyaluronidase in cleavage rate, compaction and blastocyst rate. In this context, previous study have indicated that utilizing the human recombinant hyaluronidase could bypass some problems such as the animal origin and impurity of bovine-derived hyaluronidase [8]. Characterization of HEK-PH20-1A colon demonstrated that $\mathrm{PH} 20$ coding DNA sequence was present in the cellular genome at a copy number of three copies/cell in addition to two endogenous copies. This results showed that one copy of each vectors pBud.2xPH20.attB and pBud.PH20.attB were site specifically integrated into the genome. Despite the low copy number of integrated $\mathrm{PH} 20$ donor vector in the host genome, the recombinant protein was expressed at high level in this colon. To identify the integration sites of donor vectors in the host genome, we utilized two-step inverse PCR method, which finally revealed two integration sites on the chromosomal regions of $10 q 21.2$ and 20q11.22 with a small deletion of PhiC31 pseudo attP sequence in these regions. Consistent with our results, Chalberg et al., also reported the presence of two pseudo attP sites that was used by PhiC31 integrase for transgene integration. In addition, 10q21.2 locus has been introduced as one of the most frequently used pseudo sites in human genome [33].

In summary, in the present study we developed an expression system that produced high-level functionally active rhPH20 for a long time using PhiC31 sitespecific integration of donor vectors in HEK293T cells. The clone with the highest levels of rhPH20 was characterized and the results revealed that HEK293T cells could be successfully used as an efficient human based expression system for permanent production of recombinant $\mathrm{hPH} 20$.

\section{Declarations}

\section{Acknowledgments}

This study was funded by Royan Institute for Biotechnology (grant number: 95000009)

\section{Authors' contribution}

Conceptualization, NSA, KD and LP; Data curation, NSA and LP; Formal analysis, NSA, SRV and MF; Funding acquisition, KD; Investigation, NSA, MF and RMH; Methodology, NSA, LP and SRV; Project administration, KD; Software, NSA, SRV; Supervision, KD and MHNE; Validation, MF and MHNE; Visualization, NSA and $\mathrm{RMH}$; Writing - original draft, NSA; Writing - review \& editing, KD and MHNE.

\section{Conflict of interest}

The authors have no conflicts of interest to declare.

\section{References}

1. El-Safory, N. S., Fazary, A. E. \& Lee, C.-K. Hyaluronidases, a group of glycosidases: Current and future perspectives. Carbohydrate Polymers $\mathbf{8 1 , 1 6 5 - 1 8 1}$ (2010).

2. Buhren, B. A. et al. Hyaluronidase: from clinical applications to molecular and cellular mechanisms. European journal of medical research 21, 5 (2016).

3. Csoka, A. B., Frost, G. I. \& Stern, R. The six hyaluronidase-like genes in the human and mouse genomes. Matrix biology 20, 499-508 (2001).

4. Baker, D. \& Bookbinder, L. H. (Google Patents, 2012).

5. Bookbinder, L. et al. A recombinant human enzyme for enhanced interstitial transport of therapeutics. Journal of Controlled Release 114, 230-241 (2006).

6. Frost, G. I. Recombinant human hyaluronidase (rHuPH20): an enabling platform for subcutaneous drug and fluid administration. Expert opinion on drug delivery 4, 427-440 (2007).

7. Kang, D. W., Jadin, L., Nekoroski, T., Drake, F. H. \& Zepeda, M. L. Recombinant human hyaluronidase PH20 (rHuPH20) facilitates subcutaneous infusions of large volumes of immunoglobulin in a swine model. Drug delivery and translational research 2, 254-264 (2012).

8. Evison, M., Pretty, C., Taylor, E. \& Franklin, C. Human recombinant hyaluronidase (Cumulase ${ }^{\circledR}$ ) improves intracytoplasmic sperm injection survival and fertilization rates. Reproductive biomedicine online 18, 811-814 (2009). 
9. Chen, K.-J., Sabrina, S., El-Safory, N. S., Lee, G.-C. \& Lee, C.-K. Constitutive expression of recombinant human hyaluronidase PH20 by Pichia pastoris. Journal of bioscience and bioengineering 122, 673-678 (2016).

10. Hofinger, E. S., Bernhardt, G. \& Buschauer, A. Kinetics of Hyal-1 and PH-20 hyaluronidases: Comparison of minimal substrates and analysis of the transglycosylation reaction. Glycobiology 17, 963-971 (2007).

11. Li, H. et al. Expression of a functional recombinant oleosin-human hyaluronidase hPH-20 fusion in Arabidopsis thaliana. Protein expression and purification 103, 23-27 (2014).

12. Thomas, P. \& Smart, T. G. HEK293 cell line: a vehicle for the expression of recombinant proteins. Journal of pharmacological and toxicological methods 51, 187-200 (2005).

13. Ahmadi, M. et al. Evaluating the efficiency of phiC31 integrase-mediated monoclonal antibody expression in $\mathrm{CHO}$ cells. Biotechnology progress $\mathbf{3 2}$, 1570$1576(2016)$

14. Ata-Abadi, N. S., Rezaei, N., Dormiani, K. \& Nasr-Esfahani, M. H. in Site-Specific Recombinases. 325-339 (Springer, 2017).

15. L Chavez, C. \& P Calos, M. Therapeutic applications of the PhiC31 integrase system. Current gene therapy 11, $375-381$ (2011).

16. Glover, D. J., Lipps, H. J. \& Jans, D. A. Towards safe, non-viral therapeutic gene expression in humans. Nature Reviews Genetics 6, 299-310 (2005).

17. Ata-Abadi, N. S. et al. Construction of a new minicircle DNA carrying an enhanced green florescent protein reporter gene for efficient expression into mammalian cell lines. Molecular Biology Reports 42, 1175-1185 (2015).

18. Nishiumi, F. et al. Simultaneous Single Cell Stable Expression of 2-4 cDNAs in HeLaS3 Using $\varphi$ C31 Integrase System. Cell structure and function, 0903170038-0903170038 (2009).

19. Calos, M. P. The PhiC31 Integrase System for Gene Therapy. Current gene therapy 6, 633-645 (2006).

20. Thyagarajan, B. et al. Creation of engineered human embryonic stem cell lines using phiC31 integrase. Stem cells 26, 119-126 (2008).

21. Huang, Z. et al. Recombinant human hyaluronidase PH20 does not stimulate an acute inflammatory response and inhibits lipopolysaccharide-induced neutrophil recruitment in the air pouch model of inflammation. The Journal of Immunology 192, 5285-5295 (2014).

22. De Robertis, M. et al. In vivo evaluation of a new recombinant hyaluronidase to improve gene electro-transfer protocols for DNA-based drug delivery against cancer. Cancers 10, 405 (2018).

23. Jung, Y. et al. Production of human hyaluronidase in a plant-derived protein expression system: plant-based transient production of active human hyaluronidase. Protein expression and purification 74, 181-188 (2010).

24. Dumont, J., Euwart, D., Mei, B., Estes, S. \& Kshirsagar, R. Human cell lines for biopharmaceutical manufacturing: history, status, and future perspectives. Critical reviews in biotechnology 36, 1110-1122 (2016).

25. Ghaderi, D., Taylor, R. E., Padler-Karavani, V., Diaz, S. \& Varki, A. Implications of the presence of N-glycolylneuraminic acid in recombinant therapeutic glycoproteins. Nature biotechnology 28, 863-867 (2010).

26. Lalonde, M.-E. \& Durocher, Y. Therapeutic glycoprotein production in mammalian cells. Journal of biotechnology 251, 128-140 (2017).

27. Yuan, J., Xu, W. W., Jiang, S., Yu, H. \& Poon, H. F. The scattered twelve tribes of HEK293. Biomedical and Pharmacology Journal 11, 621-623 (2018).

28. Gonzalez-Gonzalez, E. et al. Rapid and cost-effective development of stable clones for the production of anti-Ebola monoclonal antibodies in HEK293T cells. bioRxiv (2020).

29. Heuzé-Vourc'h, N. et al. Recombinant kallikrein expression: site-specific integration for hK6 production in human cells. Biological chemistry $\mathbf{3 8 7}, 687-695$ (2006).

30. Dormiani, K. et al. Long-term and efficient expression of human $\beta$-globin gene in a hematopoietic cell line using a new site-specific integrating non-viral system. Gene Therapy 22, 663-674 (2015).

31. Thyagarajan, B. \& Calos, M. P. in Therapeutic Proteins 99-106 (Springer, 2005).

32. Sivalingam, J. et al. Biosafety assessment of site-directed transgene integration in human umbilical cord-lining cells. Molecular Therapy 18, 1346-1356 (2010).

33. Chalberg, T. W. et al. Integration specificity of phage $\phi C 31$ integrase in the human genome. Journal of molecular biology 357, 28-48 (2006).

34. Singh, B. et al. Vitronectin binds to the head region of Moraxella catarrhalis ubiquitous surface protein A2 and confers complement-inhibitory activity. Molecular microbiology 75, 1426-1444 (2010).

35. Rapport, M. M., Meyer, K. \& Linker, A. Correlation of reductimetric and turbidimetric methods for hyaluronidase assay. Journal of Biological Chemistry 186, 615-623 (1950).

36. Hoebeeck, J., Speleman, F. \& Vandesompele, J. in Protocols for nucleic acid analysis by nonradioactive probes 205-226 (Springer, 2007).

37. Kent, W. J. et al. The human genome browser at UCSC. Genome research 12, 996-1006 (2002).

\section{Figures}



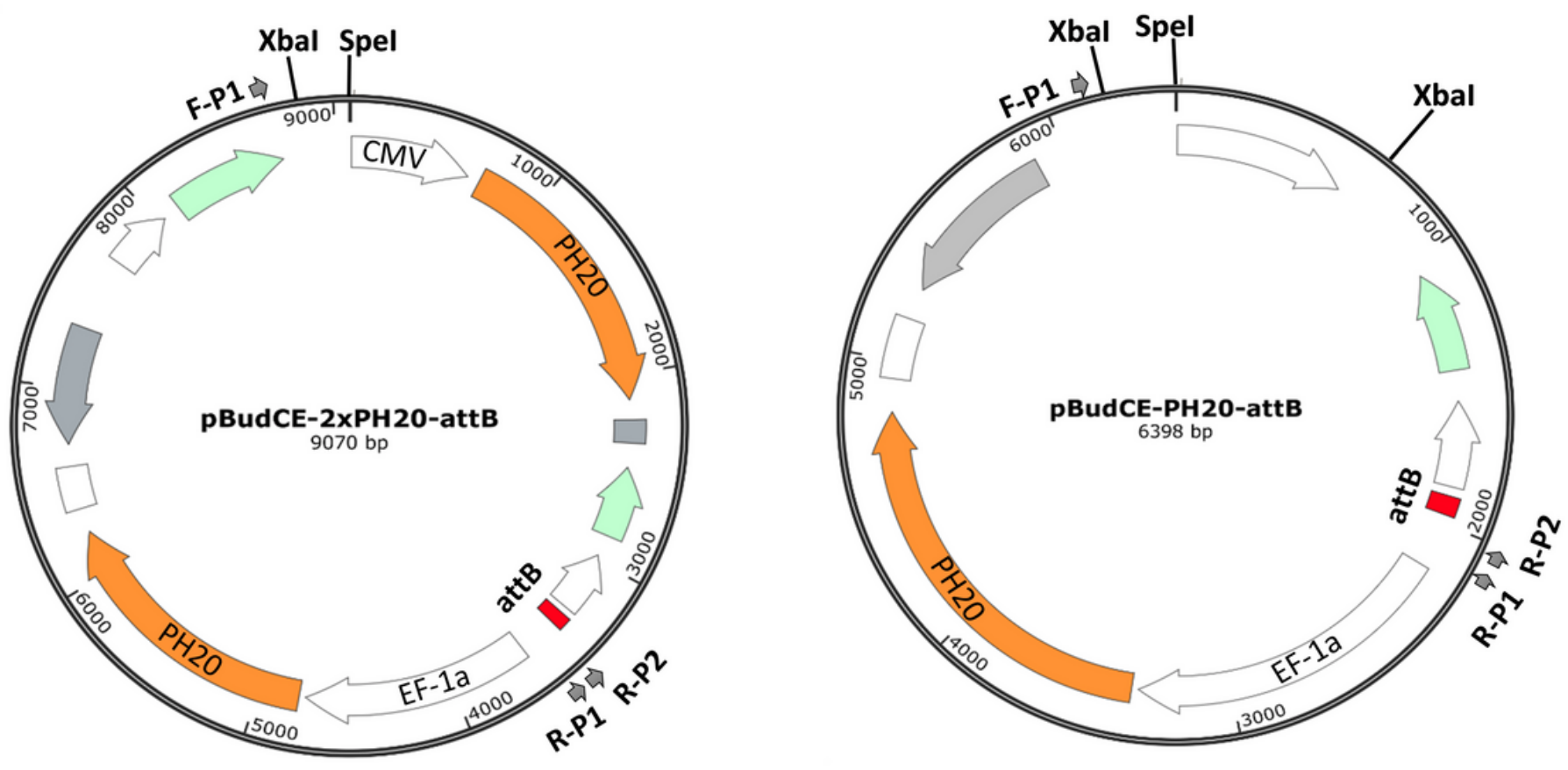

Figure 1

The maps of rhPH20 expression vectors. Schematic representation of pBUD.PH20.attB (left) and pBUD.2xPH20.attB (right) maps showed different elements in each vectors. The pBUD.PH20.attB was firstly constructed and contained human PH20 CDS under the control of EF-1a promoter and bleomycin resistance gene that confers zeocin resistance to transfected cells. The pBUD.2xPH20.attB vector was constructed using pBUD.PH20.attB as basic vector that contained more elements include the second human $\mathrm{PH} 20 \mathrm{CDS}$ under the control of $\mathrm{CMV}$ promoter and puromycin resistance gene for the second round of selection.

A

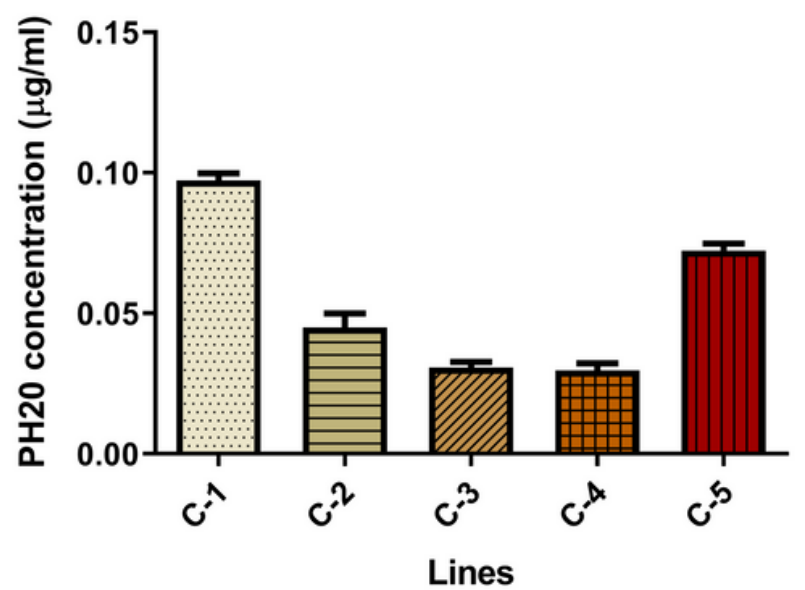

B

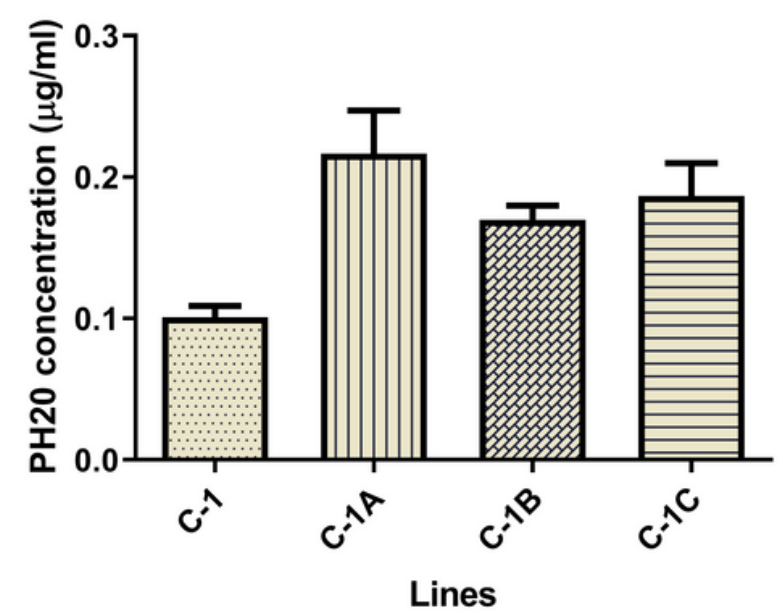

Figure 2

Absolute concentrations of the recombinant protein produced by transgenic clones. A. The $\mathrm{PH} 20$ concentrations in the culture supernatants of resulted clones after the first round of transfection with pBUD.PH20.attB. B. PH20 concentrations in the culture supernatant of the clones after the second round of transfection. In this round, the clone with the highest rhPH20 expression rate (C-1), was transfected with pBUD.2xPH20.attB. The error bars denote standard error of mean (SEM) for three independent repeats in each experiment. 


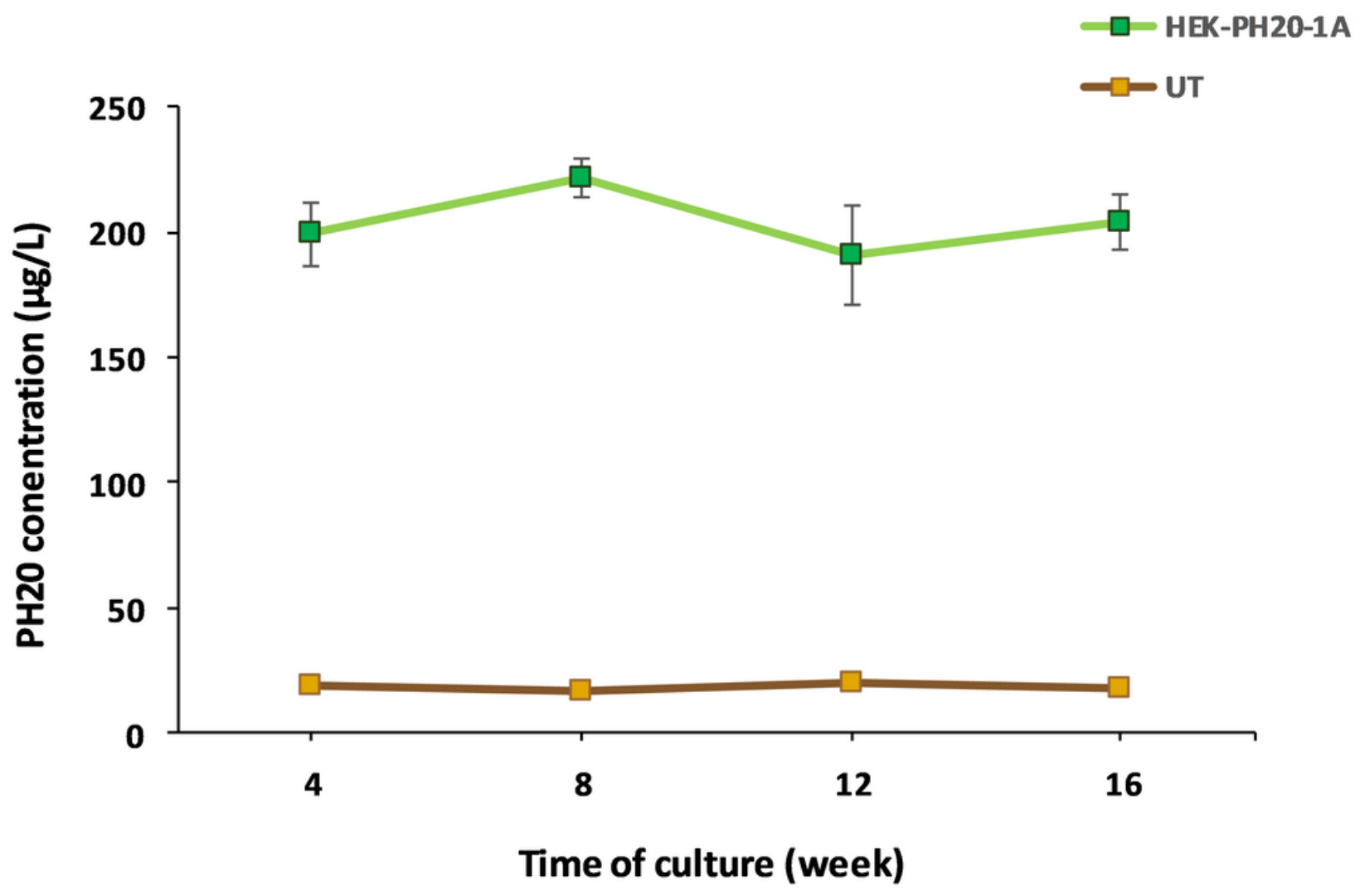

Figure 3

Monitoring the duration of rhPH20 expression by selected transgenic clones. The culture supernatant of HEK-PH20-1 A clone expressed the most amount of $\mathrm{rhPH} 20$ were monitored using human hyaluronidase ELISA kit. Significant expression of rhPH20 was evident between the tested clone and untransfected cells (UT) at each indicated time point. Moreover, the data showed that the expression level of rhPH2O by HEK-PH20-1A clone did not significantly change during a 16-week monitoring period $(P<0.05)$. 
70

\section{8 kDa}

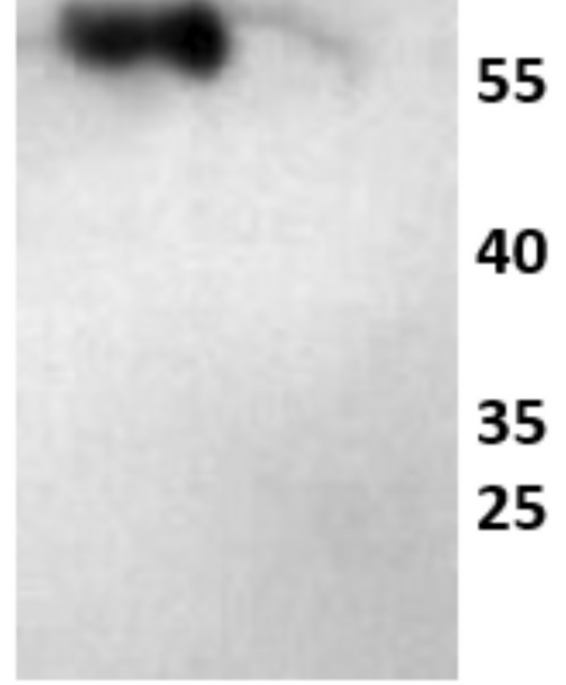

Figure 4

Western blot Analysis of purified His-tagged rhPH20 from HEK-PH20-1A culture supernatant. Approximately $58 \mathrm{kDa}$ band related to rhPH20 was resulted from western blot analysis using the Anti-polyHistidin antibody on purified rhPH20 from HEK-PH20-1A culture supernatant. 
A

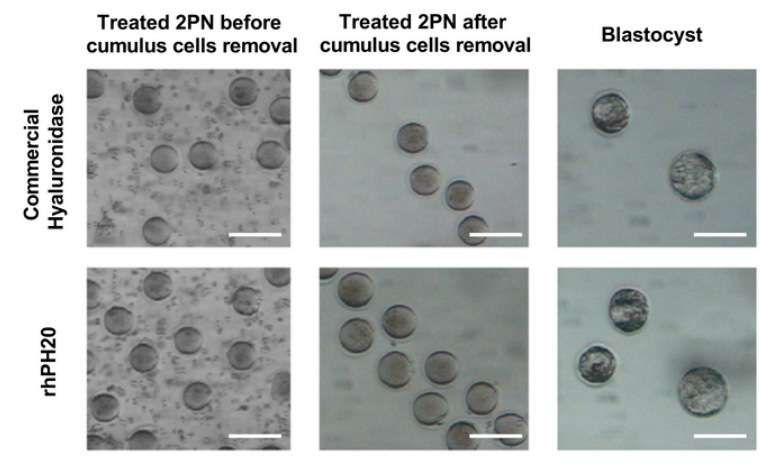

B

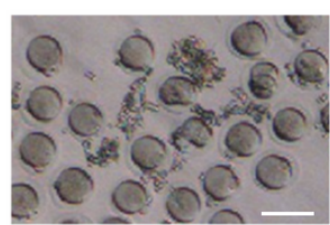

rhPH20

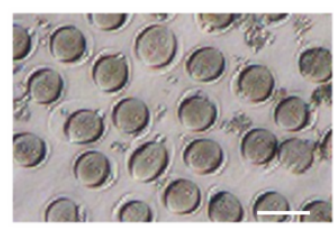

Commercial Hyaluronidase

C

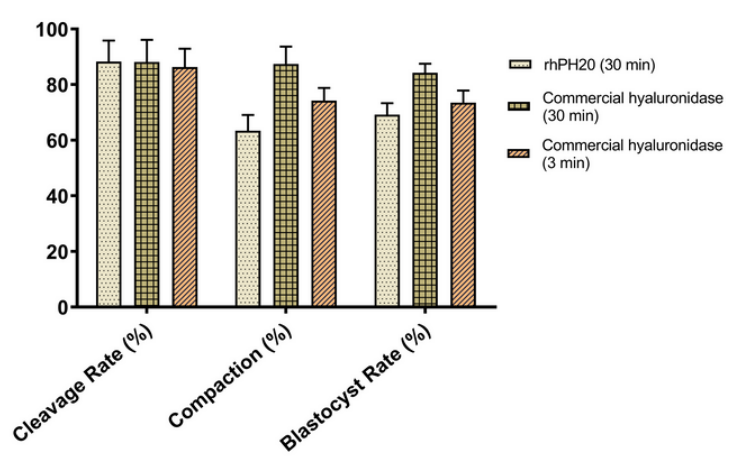

\section{Figure 5}

Assessment of functional activity of secreted rhPH20 on the removal of cumulus cells from mouse oocytes and resulting $2 \mathrm{PNs}$ embryos development. A. The mouse oocytes denoted after treatment with secreted rhPH20 from HEK-PH20-1A clone were similar to ones treated with commercially available PH20 and the quality of resulting embryos were also the same as control group. B \& C. The quality of resulting embryos did not show any significant changes in cleavage rate, compaction and blastocyst rate. Error bars indicated standard error of mean (SEM) for three independent repeats in each experiment. 
A

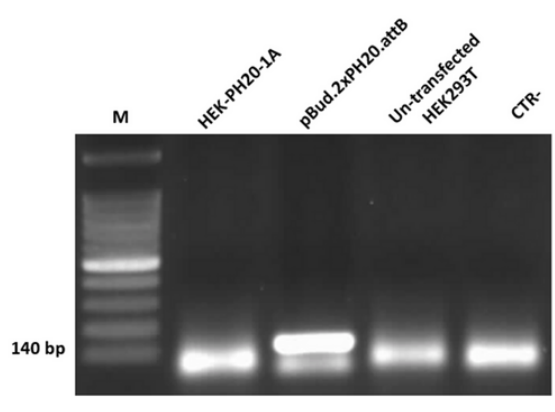

B

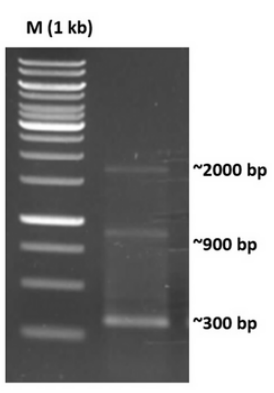

C
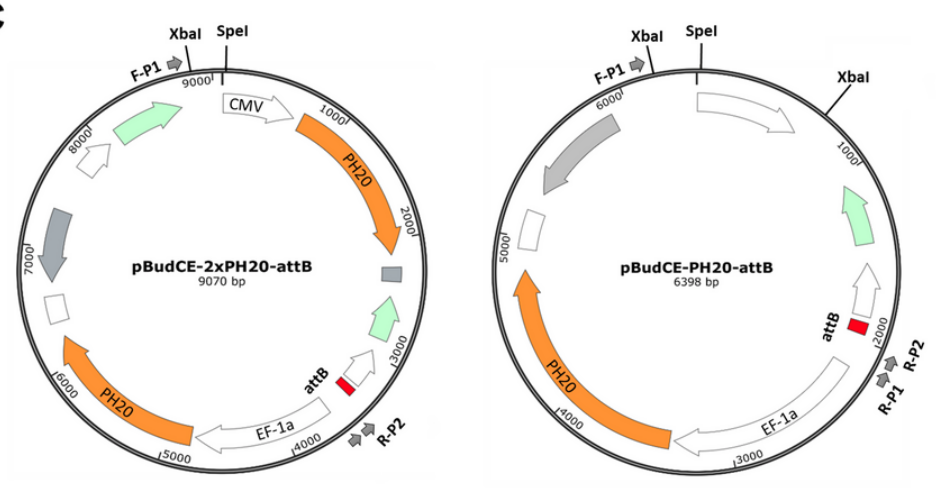

D

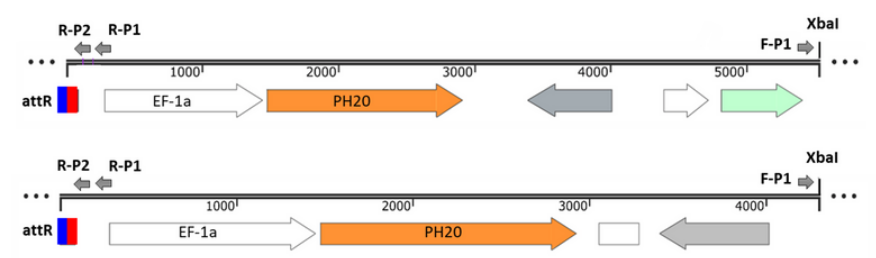

\section{Figure 6}

Site-specific integration of PH20-donor vectors in the genome of HEK-PH20 -1A clone. A. Complete sequence of attB was not amplified in the PCR reaction using HEK-PH20 -1A genome as the template. This RCR reaction confirmed a site-specific integration of the donor vector via attB site. As a positive control, we used pBud.2xPH20.attB plasmid in this PCR, which showed the expected 140 bp band. M: Marker (100 bp DNA Ladder). B. The results of nested inverse PCR on gel indicated three bands with the length of approximately 300, 900 and $2000 \mathrm{bp}$. C. Schematic representation of the restriction enzymes (Spel and Xbal) which was utilized for genomic digestion and also the sites of primer hybridization (gray arrows) on the pBud.2xPH20.attB and pBud.PH20.attB plasmids before genomic integration. D. Schematic representation of the primer attachment sites on the donor vectors after genomic integration. 

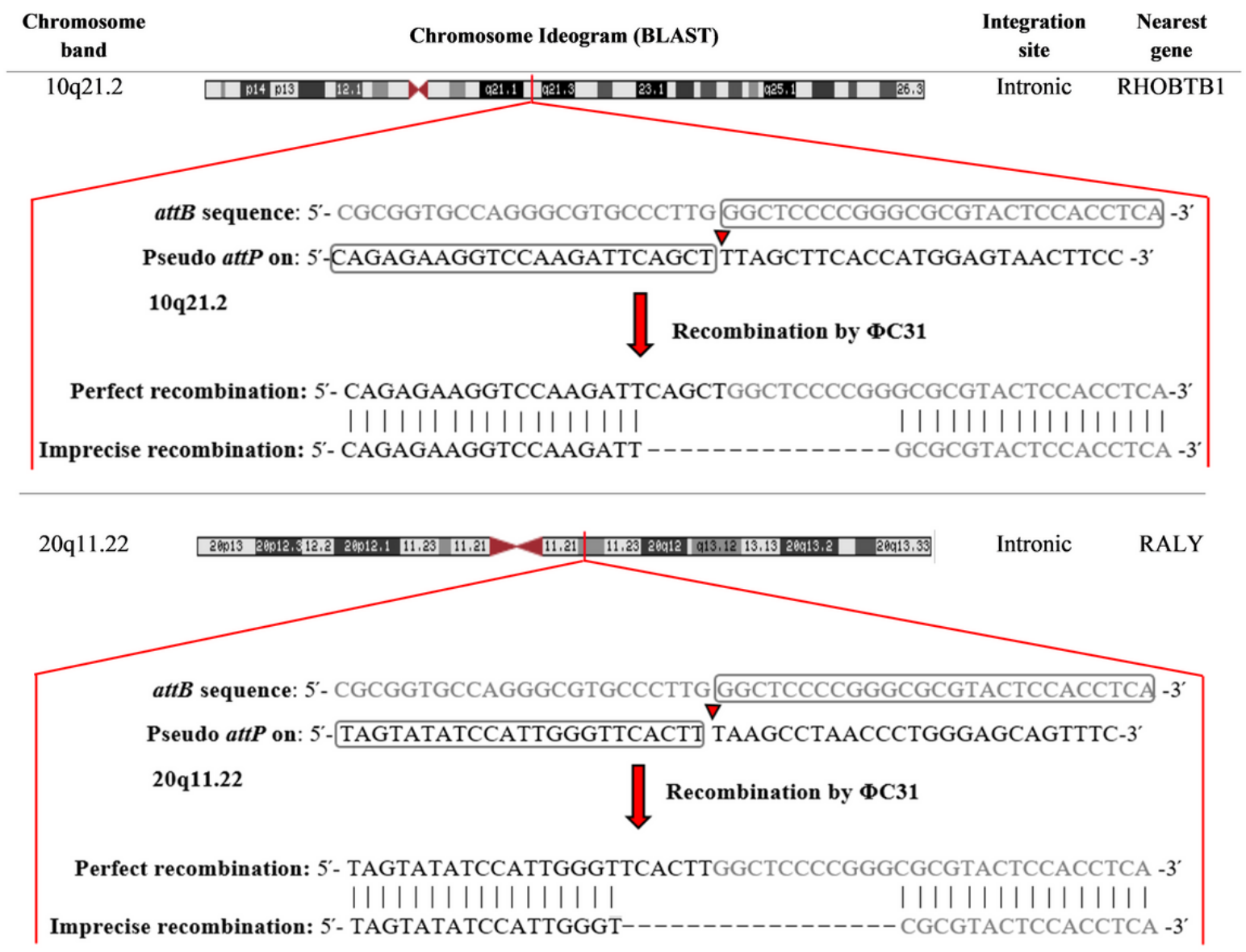

Figure 7

Identified integration sites in the genome of selected colon. Integration sites of the HEK-PH20-1A were identified by inverse PCR using genomic DNA extracted from individual clonal populations. The sequence of each integration site of donor vector were mapped to the reference human genome sequence and their respected chromosomal bands with the database at BLAT (http://genome.ucsc.edu). 